\title{
Why study the epidemiology of asthma?
}

Epidemiologists tackle questions that are complementary to those asked by other health scientists, but the questions are nevertheless distinct. Epidemiological problems are concerned with a level of organisation more complex than the individual person. By contrast, clinical and physiological problems are focused on the individual person or organ system, and cell biology and biochemistry concentrate on progressively less complex issues. Complexity does not in this context imply more or less academic sophistication, but refers simply to the extent to which problems can be isolated from more general influences for the sake of analysis. A commonplace of physiology is that experiments on isolated organs often give results different from those of experiments conducted on intact animals. Similarly, though for different reasons, the results of epidemiological studies cannot be predicted from the results of clinical or physiological studies. They contain different information and address different questions. With asthma three epidemiological questions in particular are of major importance.

\section{What sort of disease is asthma?}

The causes of disease can be classified as inherited or acquired. Most diseases are influenced by both genetic and environmental factors but the source of risk that predominates ought to determine the research strategy most appropriate for unravelling the causes of the disease. Epidemiological studies indicate with one important exception that asthma is an acquired disease determined by the environment. The only clear exception to this is atopy, which most studies show to be a genetically determined risk factor for asthma, though the precise mode of inheritance is disputed.'

Beyond this there is little evidence for the inheritance of asthma. The largest and most sophisticated analysis so far to tackle this problem directly is a segregation analysis performed by Townley and his colleagues in Omaha. ${ }^{2}$ This failed to show any genetically meaningful segregation of bronchial reactivity in families. Although this study cannot be taken as

Address for reprint requests: Dr Peter Burney, Department of Community Medicine, United Medical and Dental Schools of Guy's and St Thomas's Hospitals, St Thomas's Campus, London SE1 7EH. definitive, there is no better evidence for the alternative hypothesis that there is a further major genetic influence on asthma.

On the other hand, there is plenty of evidence that asthma is environmentally acquired. The most striking evidence for this is the large variation in asthma prevalence between similar populations living in different environments recorded in developing countries. ${ }^{3-6}$ Those in more urbanised or Westernised areas have much more asthma than those living in poorer areas, who in some cases have almost none. The observation of these differences in migrant populations $^{56}$ argues against a genetic cause, and the magnitude of the differences suggests that there are powerful environmental causes for the condition.

For this reason it appears perverse, at least to an epidemiologist, that research into the causes of asthma should have concentrated to the extent that it has over the past decade on pathological processes and mechanisms. It is as if those wishing to discover the causes of scurvy had concentrated on the pathological processes in the gums of sailors. Doubtless modern techniques could have uncovered some interesting facts in the course of such studies, but the cause of scurvy is unlikely to have been among them.

\section{What causes asthma?}

If the roots of asthma are not to be found in cells and mediators it is reasonable to ask where they are to be found. The answer is, of course, not known, but there are at least some epidemiological clues. The most striking difference in the distribution of asthma, as already mentioned, is the large variation in prevalence in developing countries. ${ }^{3-6}$ Another striking feature of the condition is the similarity in the prevalence of the condition in different social classes. Although this may seem unremarkable it is certainly unusual, and particularly so for a disease that is primarily environmental in origin. These and other features of the condition give some indication of the likelihood of competing hypotheses.

First among these at the moment is the idea that inflammation may cause asthma ${ }^{89}$ Such a notion may have gained its current popularity in part from its vagueness and the consequent ability of those studying 
any of a wide range of cells and mediators to subscribe to it. It is most unlikely to explain any major part of the distribution of asthma. Firstly, the vagueness of the hypothesis itself reduces its value. It would be hard to name half a dozen conditions of the lung that do not show some inflammatory process. They are not all accompanied by asthma. Secondly, those environmental circumstances that are generally associated with inflammatory conditions of the lung and airway do not appear to be strongly associated with asthma. If we take the presence of infection as circumstantial evidence for the presence of inflammation, children in the rural areas of developing countries have plenty of inflammatory disease in the lungs but little asthma. ${ }^{10}$ Lower social classes would be expected to have more inflammation in the lung and yet seem to have no more asthma. ${ }^{7}$ To take a further example, considerable interest has been shown in the effects of air pollution on asthma in this context. Air pollution is again relatively non-specific as the description of an exposure, but exposure to several air pollutants has been shown to produce inflammation in the airway and to affect lung function in people with asthma. The induction of reactivity by ozone in dogs has been used as a model for the pathogenesis of the condition." Nevertheless, although effects from air pollution have been detected in individuals with asthma, ${ }^{12}$ they have not been great, and the effects of very high levels of pollution on humans seem to be greater in older subjects with chronic obstructive lung disease. TokyoYokohama "asthma," which was associated with high levels of air pollution, affected almost exclusively cigarette smokers from the United States, while people with previously diagnosed atopic asthma seemed as likely to get better as worse on going to the affected area. ${ }^{13}$ Finally, the populations of some very severely polluted environments seem to have virtually no asthma. ${ }^{4}$

If inflammation is to be a helpful concept in explaining the aetiology of asthma, it needs to be endowed with greater specificity of meaning. Even then, however, it may be as useful to say that asthma is caused by inflammation as it is to say that pneumonia, sarcoidosis, or fibrosing alveolitis are caused by inflammation. Meanwhile we require alternative hypotheses to explain the distribution of the condition in terms of its environmental determinants.

\section{Is the management of asthma effective?}

There are two distinct measures of whether a particular method of managing a condition is adequate. Epidemiologists distinguish between "efficacious" treatments, which are those that under ideal circumstances achieve what they are intended to, and "effective" treatments, which have a beneficial effect on the population as a whole. ${ }^{14}$ Randomised controlled trials $\vec{\Rightarrow}$ are used to assess what happens to subjects given a treatment under experimental conditions. The circumstances of treatment in such trials are tightly con- $\frac{\bar{O}}{\bar{D}}$ trolled and the subjects selected are often those most $\frac{\rho}{\Phi}$ likely to comply with the regimen; they may even be $\stackrel{0}{2}$ those most likely to respond to the treatment. The $\approx$ randomised clinical trial has become the cornerstone $\vec{\circ}$ of modern practice in assessing treatment; but there are aspects of management that cannot be assessed in $\vec{\omega}$ this way, and the overall effect of management cannot be predicted from the results of randomised trials $x$ alone.

Firstly, randomised trials are rarely large or exten- $\dot{\sigma}$ sive enough to test whether a particular effect will be $\vec{N}$ found in every group of subjects, or to exclude the possibility of a rare adverse effect. Secondly, the response to the drug will be influenced by the selection $\overrightarrow{\vec{C}}$ of patients to take the drug. A drug that has a favourable balance of therapeutic to unwanted effects $\stackrel{\oplus}{\Phi}$ in subjects selected, in the first instance, because they $\vec{\oplus}$ are believed to be the sort of patients who will benefit $\varphi^{\infty}$ may show an adverse ratio of benefits to costs if the use of the drug extends to less ill patients or to those less suited to that treatment. A drug that is highly efficacious in a trial may well have disappointing or even adverse effects on the population as a whole.

The recent increase in mortality from asthma in $\overrightarrow{7}$ several countries, including the United Kingdom, ${ }^{15} \frac{3}{3}$ and the wide variation in reported asthma mortality between areas and countries ${ }^{1617}$ inevitably raise questions concerning the overall effectiveness of modern treatment of asthma. The prescription of efficacious treatments for asthma has been rising steadily ${ }^{18}$ while $\underset{x}{\stackrel{0}{\nu}}$ mortality from other "preventable" causes of death has been falling consistently, both in the United 3 . Kingdom and elsewhere. ${ }^{19}$ Perhaps the prevalence of $\%$ asthma is increasing, and perhaps the prevalence varies considerably from one country and from one $\mathrm{O}$ part of a country to another. These are epidemiological questions that have yet to be ans-o wered. If differences in prevalence do not account for $\stackrel{\bar{N}}{\bar{N}}$

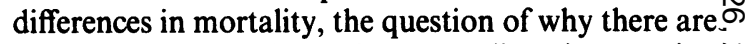
such wide variations in mortality is another $N_{0}$ epidemiological problem of great interest. The development and testing of adequate medication is only one aspect of a much more complex problem of providing an adequate service for the treatment of patients with asthma.

\section{Why study the epidemiology of asthma?}

Epidemiology is often portrayed in clinical textbooks $\frac{?}{\mathbb{1}}$ as a descriptive discipline of the sort condemned $a_{0}$ century ago by Claude Bernard. Epidemiologists, by contrast, spend most of their time in testing hypoth-o응 
eses. The methods used are principally, though not exclusively, non-experimental and this determines some of the characteristic strengths and weaknesses of the method.

Experimental design is well adapted to showing that an effect can be achieved from a particular exposure. Random allocation of large numbers to different exposures and other safeguards are designed to make alternative explanations of the results unlikely. But to show by experiment that an exposure can achieve an effect says nothing of what happens outside the laboratory. To show that a duke can be drowned in a butt of Malmsey does not give dukes in general any realistic estimate of the risk they take when entering a cellar. In the same way showing that inhalation of platelet activating factor, or even allergen, increases reactivity in the airway does not show how much risk this poses either to asthmatic or to non-asthmatic individuals in the world at large.

Purely observational studies are by contrast designed to identify those factors that are associated with natural variation in the subject of concern. Their principal weakness resides in the possibility that conclusions may be influenced by confounding sources of variation. A confounding variable is one that is associated with both the supposed cause and the supposed effect. It is possible, for instance, though not now likely, that the association observed between dietary sodium and the bronchial response to histamine was due to the confounding effect of food additives. ${ }^{20}$ These might cause increased bronchial reactivity and are very likely to be associated with a high sodium diet as both salt and additives are found in considerable quantities in preprepared food.

Not surprisingly, confounding has become a major interest of epidemiologists. Apart from statistical methods of dealing with potential confounders some distinction can be made between a true causal association and one due to confounding by examining the characteristics of the association itself. ${ }^{21}$ Those that are consistent in several studies, strong, statistically significant, and specific to the particular disease and risk factor are more likely to be true causal associations, as are those associations where there is evidence for the cause preceding the supposed effect, those that show an increasing effect with increasing dose, and those that are in accord with current biological theory. None of these characteristics, however, is an entirely reliable guide and the final vindication of epidemiological conclusions about the causes of illness may rest on the experimental evidence of what happens when the alleged cause is removed. Such experiments, however, are not dependent either in design or in interpretation on prior knowledge of the mechanisms of disease. Scurvy can be shown experimentally to be associated with lack of fresh fruit and vegetables without any knowledge of the existence of vitamin $\mathrm{C}$, let alone its mode of action.

For over a decade now research on the aetiology of asthma has concentrated on the expensive investigation of pathogenetic hypotheses detached from any credible environmental explanation of the disease. It is perhaps time to take more seriously the evidence that asthma is in large measure an acquired condition. The mechanism of a disease is unlikely to be discovered before its cause, and at a time of apparently dwindling resources investment in the epidemiology of asthma should pay good dividends.

PETER BURNEY

Department of Community Medicine United Medical and Dental Schools of Guy's and St Thomas's Hospitals

St Thomas's Campus London SE1 7EH

\section{References}

1 Marsh DG, Meyers DA, Bias WB. The epidemiology and genetics of atopic allergy. $N$ Engl J Med 1981; 305:1551-9.

2 Townley RG, Bewtra A, Wilson AF, et al. Segregation analysis of bronchial response to methacholine inhalation challenge in families with and without asthma. $J$ Allergy Clin Immunol 1986;77:101-7.

3 Godfrey RC. Asthma and IgE levels in urban and rural communities in the Gambia. Clin Allergy 1975;5:201-7.

4 Anderson HR. Respiratory abnormalities in Papua New Guinea children: the effects of locality and domestic wood smoke. Int J Epidemiol 1978;7:63-72.

5 Van Niekerk CH, Weinberg EG, Shore SC, Heese H de V, Van Schalkwyk DJ. Prevalence of asthma: a comparative study of urban and rural Xhosa children. Clin Allergy 1979;9:319-24.

6 Waite DA, Eyles EF, Tarkin SL, O'Donnell TV. Asthma prevalence in Tokelnan children in two environments. Clin Allergy 1980;10:71-5.

7 Anderson HR, Bland JM, Peckham CS. Risk factors for asthma up to 16 years of age: evidence from a national cohort study. Chest 1987;91:127-30S.

8 Dolovich J, Hargreave $\mathrm{FH}$. The asthma syndrome: inciters, inducers and host characteristics. Thorax 1981;36:641-4.

9 Holgate S, Beasley R, Twentyman OP. The pathogenesis and significance of bronchial hyperresponsiveness in airway disease. Clin Sci 1987;73:561-72.

10 Cookson JB. Prevalence rates of asthma in developing countries and their comparison to those in Europe and North America. Chest 1987;91:97-103S

11 Lee LY, Blecker ER, Nadel JA. Effect of ozone on bronchomotor response to inhaled histamine aerosol in dogs. J Appl Physiol 1977:43:626-31.

12 Whittemore AS, Korn EL. Asthma and air pollution in the Los Angeles area. Am J Publ Health 1980;70: 687-96.

13 Phelps HW, Koike S. "Tokyo-Yokohama asthma": the 
rapid development of respiratory distress presumably due to air pollution. Am Rev Respir Dis 1962;86:53-63.

14 Last JM, ed. A dictionary of epidemiology. Oxford: Oxford University Press, 1983.

15 Burney PGJ. Asthma mortality in England and Wales: evidence for a further increase 1974-84. Lancet 1986;ii:323-6.

16 Charlton JRH, Hartley RM, Silver R, Holland WW. Geographical variation in mortality from conditions amenable to intervention in England and Wales. Lancet 1983;i:691-6.

17 Council of the European Communities. European Community atlas of avoidable death. Oxford: Oxford University Press and Council of the European Com- munities, 1988. (EEC Health Services Research Series, No. 3.)

18 Keating G, Mitchell EA, Jackson R, Beaglehole R, Rea $H$. Trends in sales of drugs for asthma in New Zealand, Australia and the United Kingdom, 1975-81. Br Med J $\overline{\bar{C}}$ 1984;289:348-51.

19 Charlton JRH, Velez R. Some international comparisons in mortality amenable to medical intervention. $\mathrm{Br} \mathrm{Med}$ $J$ 1986;292:295-301.

20 Burney PGJ, Britton JR, Chinn S, et al. Response to. inhaled histamine and 24 hour sodium excretion. $\mathrm{Br} \overrightarrow{\vec{G}}$ Med J 1986;292:1483-6.

21 Hill AB. A short textbook of medical statistics. London: Hodder and Stoughton, 1977. 